\title{
Theory Into Practice: Applying Managerial Accounting Tools To Solve A Real-World Dilemma
}

Frederick C. Teufel, Jr., Saint Joseph's University, USA Joseph Larkin, Saint Joseph's University, USA

\begin{abstract}
Many high school and college graduates that have excelled in their sport at either or both levels are not able to maintain their edge once they finish matriculating and/or enter the work force. The demands on their time coupled with the gradual deterioration of their fitness and athleticism that comes along with life beyond the academic setting reduces them to weekend warrior status. But the desire to be part of a winning team and pushing the limits of competition do not end there. In fact, many former athletes volunteer to take on leadership roles off the field that are connected to the sport that they excelled at in school. To some, those roles can be no more than administrative functions that fail to scratch the competitive itch of the former athlete. Eric Miller, an accomplished college soccer player and recent business school graduate had a different idea when he volunteered to take on the team manager role of a local youth sports team. Eric looked at this as an opportunity to apply what he learned in his business classes about strategy development and managerial accounting. Eric started his analysis by looking at the team's financial situation. Eric then framed out what he believed to be the areas that he needed to address and is seeking your assistance in helping with his analysis so that he can meet his goal of creating the most value for his team.
\end{abstract}

Keywords: Budget; Cost; Value; Analysis; Strategy

\section{INTRODUCTION}

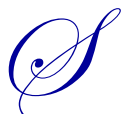
oon after his graduation from college, Eric Miller returned to the community that he left behind. In his younger years, Eric was a star soccer player for his local youth sports team. Eric had fond memories of his days playing soccer at the local club fields with the friends and neighbors that he grew up with and was anxious to return to those fields as a spectator. So one early fall Saturday afternoon, Eric wandered down to the community fields to watch one of his neighbor's children play a soccer game. Eric was surprised to find that the club team he once played for and that had achieved much success, had barely enough players to field a team and were clearly overmatched that day. After the game, Eric approached the coach to introduce himself as a former club player and learned from the coach that the club was experiencing significant challenges and that "if things didn't improve," the coach predicted," the team" which had provided opportunities for local teenagers to compete for generations, "would have to fold."

Later that day, Eric reached out to the club president in an effort to understand what was happening and why. His conversation with the club president was enlightening. In summary, this is what Eric heard:

The team has had some success in the league they have been competing in but have reached an inflection point. The team's star players were recruited away by so-called premier programs that tout "exposure to college recruiters," "paid professional coaching," and "state-of-the-art venues" as reasons for players to abandon their existing team and sign on with them. And, if that wasn't enough, this year those premier programs began casting their nets even beyond the team's star players so that they can field $B$ and $C$ level teams. As an example he said, a player whose parent was loyal to the club and served as the team manager was recruited away and that the team had been operating without a manager for several weeks. 
Eric, seeing this as an opportunity to give back to his local community, volunteered to step in as the team's manager. His offer was immediately and enthusiastically accepted by the club president. Eric's next stop was to revisit the team's coach to learn more about the role of the manager. In that conversation, he learned that the previous manager had done an excellent job in making sure that all of the administrative elements of the role had been attended to with aplomb and that if he could just get the team's records and forms caught up the coach would be pleased. However, as a recent graduate of a respected business school, Eric knew that there was a lot more that he could do.

So now having gotten input from the coach and club president, he began conducting interviews with other key stakeholders, including current and former parents and players, league officials, and community members and based on what he heard, drafted his team's mission statement:

To provide an enriching experience to community youth by maintaining an organization that offers the opportunity to play soccer at a highly competitive and affordable level.

Eric shared his mission statement with key stakeholders who enthusiastically ratified it and off Eric went to identify and prioritize his key challenges and opportunities. He quickly realized that his first priority would have to be to improve the team's financial picture. As a start, Eric needed to get his arms around the current costs and prepare a budget for the upcoming season.

\section{The Costs of Maintaining the Status Quo}

\section{Pricing and Funding}

The team operates on an "at cost" basis. Each year all of the team's costs are passed through to the players. The team's manager requests player payments throughout the year in an effort to break even. The current cost structure does not provide for any form of margin. The team has not pursued any funding opportunities such as soliciting sponsors, obtaining grants or canvassing the local community for contributions that might be available to help defray operating costs.

\section{Membership Fee}

The team currently pays the club a membership fee assessed to each player at the rate of $\$ 300$ per player per season. The membership fee compensates the club for the use of the fields and administration, including insurance. Although the club has been able to keep the fee relatively flat for the past five years, increases in field maintenance and rental costs, and administrative and web support costs coupled with the declining number of registrations will surely drive up per player registration fees. In fact, per player fees are expected to increase by $\$ 50$ or $17 \%$ in the upcoming year.

\section{Professional Coaching Fee}

To remain competitive with other travel soccer programs in the area, the club no longer uses parent volunteers to coach their travel soccer teams. Instead, professional coaches are hired to train several teams during the course of the season. These coaches are independent contractors and negotiate compensation amounts with the club on an individual basis. Under the current arrangement, teams are required to pay the club a fee of $\$ 750$ per-player per season year for this service. Teams are assigned a coach by the club and do not have the opportunity to choose a particular coach. Further, the number of training sessions has no effect on the per-player fee. The number of training sessions for a team may vary from once-a-week to as many as three-times-per-week, yet the per-player assessment remains the same.

\section{Uniforms}

Players are required to purchase a home and away uniform (kit), a "special" uniform for tournaments, a training outfit and a warm up suit. The standard home and away kit includes socks, shorts and a jersey at a cost of $\$ 150$ per kit for a total of $\$ 300$ per player for both packages. The club charges $\$ 150$ for the warm-up suit and $\$ 100$ for the 
training kit bringing the total to $\$ 550$ per player. Since most players purchase at least two training kits, the total cost for uniforms can easily reach or exceed $\$ 650$ per player.

\section{League Fees}

The club is affiliated with the Rydal Travel League (RTL) and therefore each club team is required to register and compete in the RTL as their primary league. The RTL regular season begins in August and ends in the middle of November. Teams are free to participate in any other league as a secondary league during that time period and in any other league as a primary league in the winter, spring or summer seasons. Each team pays $\$ 2,500$ to the club to register for the RTL fall season. In addition to the fall season, the team typically registers for a winter indoor league and a spring league, as well. The registration fee is $\$ 2,000$ for the winter and $\$ 1,500$ for the spring. These fees are paid directly to the leagues.

\section{Game Official Fees}

Each team is responsible for paying referee game fees. The fees vary by age group. Three officials are assigned for each match. For the current age group, the center official receives $\$ 95$ per game and the two line assistant referees receive $\$ 42.50$ per game. The total cost is split evenly between the two teams. Each team is responsible for $1 / 2$ the fees as the other team is responsible for the other half. The comprehensive schedule for the year includes: Fall -10 games; Winter -8 games; Spring -6 games.

\section{Tournaments}

When Eric looks back at his travel soccer experience, he recalls that his biggest thrills and most memorable experiences came from his team's participation in travel soccer tournaments. Often, college coaches scout and recruit prospective student-athletes at the higher-level events. So, these tournaments offer outstanding exposure for players who aspire to compete at the college level.

As a matter of policy, the club requires that each team compete in the tournament sponsored by the club in June of each year. The club also provides each team with a list of "sanctioned" tournaments. From this list, each team is free to pick and choose the tournaments that they will play. Many teams will opt for the higher level tournaments for the exposure to the college recruiters.

Registration fees range from $\$ 550$ to $\$ 1200$ per tournament. In addition to the application fee (which average $\$ 750$ per tournament), tournament selection is affected by many variables including tournament rankings, travel distance, length of tournament, length of required overnight stay (averages two nights) and accommodation cost (averages $\$ 200$ per night). Families are expected to pay for their own gas and accommodations; however, the team pays for players' hotel accommodations. Two players are assigned to a room. Players and guests are expected to provide for their own meals. In addition, the club must pay the coach $\$ 200$ in addition to the annual coaching fee they pay to the club for each away tournament.

\section{Field Rentals}

Soccer has long been considered the World's most popular game and its fascinating history has been recounted in volumes. One of the reasons the sport is so universally played is that minimally it requires an individual to have only a sphere and time in order to train. Of course, and although mastery of the skills required to play the game doesn't require significant resources, like so many other major sports the game has evolved beyond the past-time it once was to a major business and spectator sport at the highest levels of competition. That evolution has created demand for greater resources. Perhaps the greatest demand and scarcest resource is the fields/facilities that are required to train and play competitive games as a team. So, it should be no surprise then that field/facility costs are a significant financial consideration. Moreover, since not all fields/facilities are of equal value, the identification of and selection of fields/facilities are a key component of a team's value proposition. Recognizing that, Eric developed the following Tier structure to differentiate field/facility options. 
Tier 1: Higher quality artificial surfaces that can be used in almost all weather conditions. Dimensions and conditions are appropriate for the intended use.

Tier 2: Lower quality artificial surfaces and high quality natural grass surfaces that may be restricted because of weather conditions. Dimensions and conditions are appropriate for the intended use.

Tier 3: Lower quality natural surfaces that may be restricted because of weather or other issues related to maintenance and general upkeep. Dimensions are appropriate for the intended use.

Tier 4: Use at your own risk. The field/facility is not well maintained, may not be the dimensions and conditions needed for the intended use.

The club has a perpetual lease with the local township for $\$ 1$ per year for the use of a township owned property. The lease is for 99 years and is the only real asset that the club holds. The property comprises hundreds of acres of open space that the club uses as a practice facility and to host games. The club has carved out eight fields for use by its members. The club is responsible for maintaining the fields. Club membership fees paid by each player are used to pay for the maintenance of the fields. The fields are kept in excellent condition (considered a Tier 2 facility) and the club allots each team certain practice and game times. Of the eight fields that are available, only two meet the required dimensions for the team's age group. The club has been very generous and accommodating to the team, such that there have been no scheduling issues to speak of. However, the fields are all grass and can only be used from August to November and April to June ... and even then, only when there is no rain in the forecast. Although the team was able to work around those restrictions in the past, the team's primary training and league seasons run from December through July. Therefore, the team has to find artificial turf fields for practices and to host games when the club fields are shut down. For winter training, the team pays the club $\$ 300$ per use to use a local high school gym, a tier 3 facility. For outdoor training, the club charges the team for the use of artificial turf fields they lease from local high schools at a cost of $\$ 250$ per session (Tier 1 facilities). Eight matches are scheduled for the winter (four home games/four away). Fourteen training sessions are held during the winter months, each requiring the rental of artificial turf fields. In addition, the team trains 14 times indoors from November to early March. Lastly, the team rents an artificial turf field to conduct spring training since the club fields are not open until mid April and to cover the training sessions that club fields are closed because of rain. So an additional 10 outdoor (artificial turf) field rentals are required.

Other relevant information:

- Although the club maintains a website that each team uses to communicate key club events and activities and facilitate the collection of fees, each team subscribes to a team management utility to communicate with players and parents and to organize events. The team pays a subscription fee of $\$ 420$ per year charged at the rate of $\$ 35$ per month.

- The team may roster as many as 22 players. Since only 11 players are on the field at a time, the current coach has capped the roster at 15 to maximize individual playing time. For tournaments, the team has used non-rostered "guest players" to supplement the roster. The team has never had the full 15 man roster at any one game. Guest players are not asked to make a financial contribution.

- A survey conducted by the State Youth Soccer Association revealed that the cost per player (CPP) to play for a year round soccer team ranged from $\$ 500$ to $\$ 7,500$. Teams were further broken down into categories based on certain criteria. The survey found that regardless of the category, on average, $30 \%$ of the fees were used to pay for membership costs (including website fees and field rentals; $30 \%$ were used to pay for coaching; $15 \%$ were used to pay for uniforms and $25 \%$ were used to pay for league and tournament fees (including referee fees). The following is a list of categories and their respective ranges of per player fees:

- Academy Programs (Player Development/Team Competition is not emphasized) - Players had to be fully committed to the program for the full year and are not permitted to play any other sport nor play with their school soccer team. Teams are assigned professional coaches and compete in only nationally ranked tournaments. Teams train and compete in only Tier 1 facilities. Costs 
Range is $\$ 5,000$ to $\$ 7,500$ per year plus travel expenses. Most Academy Programs are associated with sponsors that helped defray costs.

- Premier Programs (Individual Player Development and Team Competition are equally promoted)

- Players have to commit to a full year and prioritize team activities over any other sports activities. Teams are assigned professional coaches and compete in both nationally ranked as well as regionally ranked tournaments. Teams train and compete in mostly Tier 1 facilities but offered at least Tier 2 facilities for home games. Cost Range is $\$ 3,500$ to $\$ 5,500$ plus travel expenses.

- Select Programs (Team Competition is emphasized and Individual Player Development is ancillary) - Players have to commit to a full year and prioritize team activities over any other sports activities during the team's primary season. Teams are assigned professional coaches and compete mostly in local tournaments but occasionally play in nationally ranked or regionally ranked tournaments if qualified. Teams train and compete in Tier 1, Tier 2 and sometimes Tier 3 facilities. Home fields are either Tier 2 or 3 facilities. Cost Range is $\$ 2,000$ to $\$ 5,000$ plus travel expenses.

- Club Programs (Team Competition is de-emphasized, Individual Player Development is a byproduct, Community-based). Players commit to the primary season. Teams are assigned professional coaches and competed only in local tournaments. Teams train and compete in Tier 2, Tier 3 and Tier 4 facilities and have to have a designated home field. Cost Range is $\$ 500$ to $\$ 1,200$ plus travel expenses, if any.

- The team budgets $\$ 1,000$ per year for miscellaneous expenses not originally anticipated.

- Eric's brainstorming sessions with other coaches, administrators and parents and web searches identified several potential sources of funding that could be used to defray player/team costs, including local business and national sponsorships; grants; corporate donations; individual contributions and team run events and programs (skills clinics, car washes, tournament administration). However, as excited as Eric was to pursue those funding sources, he decided that developing funding sources would have to be a "day 2" activity and that he should focus on the cost side of the budgeting equation. After all, Eric thought to himself, those efforts would consume more time than he was willing to volunteer given that he had other professional and personal commitments. Eric's preliminary assessment of funding sources resulted in the following conclusions:

- A local soccer equipment and uniform supplier agreed to rebate $20 \%$ of the price paid to the store to the team for soccer equipment purchased by a player or member of the player's family and donate $\$ 50$ per player if the team agreed to display the stores banner at tournaments and imprint the store's logo on player's jerseys.

- Skills clinics had become very popular in the area and could be conducted year-round. Skills clinics typically yielded between $\$ 500$ to $\$ 1500$ each depending on the level of training provided and the location. Eric thought that it would be reasonable to expect the team to put on at least 1 skills clinic per quarter.

- It was not uncommon for teams to conduct car washes from time to time. Local car servicing centers in the area were known to allow charities to use their facilities free of charge since motorists stopping for a car wash often purchased gas at the same time. Car washes usually generated between $\$ 200$ and $\$ 600$ depending on the timing and conditions.

- Tournament administration requires a significant effort on the part of team players and parents with tournament revenues coming from team fees, sponsors and food service rebates. Eric learned that the team's current club generated between $\$ 25,000$ and $\$ 35,000$ annually of net revenue from their spring tournament. Eric realistically estimated that his team was capable of running a tournament sizeable enough to generate $\$ 7,500$ to $\$ 10,000$ per year.

- Eric rationalized that his team would not be a good candidate for grants, corporate, individual or community donations given the team's demographic profile and financial status of most of the current team members' families. 


\section{CASE QUESTIONS}

1. Prepare an estimate of the team's costs for the current year using the information provided. How much will each player (assuming the current roster of 15 players) have to pay in order for the team to cover costs?

2. Determine how much each player will have to pay if referees fees are increased by $10 \%$, club fees increase by $5 \%$, the team plays 9 tournaments instead of 8,2 of the 9 tournaments are local.

3. How much of the team's cost go to the club? How would you evaluate the cost/benefit of club membership? What additional information would you need from the club to do that evaluation?

4. Evaluate the short term options and alternatives that Eric could implement to get the per player fees more in line with the midpoint of the Select Program range.

5. Although Eric did not pursue sources of funding or revenue initiatives, what impact might the revenue opportunities outlined above have on the team's financial future? Would they help the team achieve its mission? If so, how?

\section{TEACHING NOTES}

This case is appropriate for students taking a Managerial Accounting course. At its core, the case can be used to help students understand how to prepare a budget, differentiate fixed and variable costs, direct vs. indirect costs and allocation bases. At another level, this case can be used to evaluate strategic options in the context of meeting financial targets and goals. Students generally enter college with very little understanding of how much of a factor cost is in most choices that adults have to make. Students should be able to prepare a budget for the current year using the information from the case and assuming the status quo. Although the case and the budgeting process is focused mainly on cost behaviors, drivers and outcomes and the cost vs value proposition, the information provided on sources of funding and revenues could be used to stimulate some discussion about the limitations of the team's current business model and get the students to realize that although cutting costs may be the most direct and easiest way to improve the financial picture in the short run, identifying new sources of revenue and diversification of revenue sources may have a sustainable benefit in the long run.

Table 1. Current Annual Budget

\begin{tabular}{|c|c|c|c|c|c|}
\hline Costs & Cost Driver & Number & Cost Per & Total & PerPlayer \\
\hline Membership Fee & Players & 15 & $\$ 500$ & $\$ 7,500.00$ & $\$ 500.00$ \\
\hline Coaching Fee & Players & 15 & $\$ 1,000$ & $\$ 15,000.00$ & $\$ 1,000.00$ \\
\hline \multicolumn{6}{|l|}{ Uniforms } \\
\hline Home/Away & Players & 15 & $\$ 300$ & $\$ 4,500.00$ & $\$ 300.00$ \\
\hline Training Kit & Players & 15 & $\$ 100$ & $\$ 1,500.00$ & $\$ 100.00$ \\
\hline Warm Up & Players & 15 & $\$ 150$ & $\$ 2,250.00$ & $\$ 150.00$ \\
\hline \multicolumn{6}{|l|}{ League Fees } \\
\hline Fall & Per Season & 1 & $\$ 2,500$ & $\$ 2,500.00$ & $\$ 166.67$ \\
\hline Winter & Per Season & 1 & $\$ 2,000$ & $\$ 2,000.00$ & $\$ 133.33$ \\
\hline Spring & Per Season & 1 & $\$ 1,500$ & $\$ 1,500.00$ & $\$ 100.00$ \\
\hline Summer & Per Season & 0 & - & $\$ 0.00$ & $\$ 0.00$ \\
\hline Game Official Fees & Games & 24 & $\$ 95$ & $\$ 2,280.00$ & $\$ 152.00$ \\
\hline Tournament Fees & Tournaments & 8 & $\$ 750$ & $\$ 6,000.00$ & $\$ 400.00$ \\
\hline Hotel Costs & Overnight Tournaments & 3 & $\$ 3,200$ & $\$ 9,600.00$ & $\$ 640.00$ \\
\hline Coaching Fee & Overnight Tournaments & 3 & $\$ 200$ & $\$ 600.00$ & $\$ 40.00$ \\
\hline Website Maintenance & Per annum & 1 & $\$ 420$ & $\$ 420.00$ & $\$ 28.00$ \\
\hline Miscellaneous & Total & 1 & $\$ 750$ & $\$ 750.00$ & $\$ 50.00$ \\
\hline Indoor Field Rental & Per use & 14 & $\$ 300$ & $\$ 4,200.00$ & $\$ 280.00$ \\
\hline \multirow[t]{2}{*}{ Outdoor Turf Rental } & Per use & 28 & $\$ 250$ & $\$ 7,000.00$ & $\$ 466.67$ \\
\hline & & & & $\$ 67,600.00$ & $\$ 4,506.67$ \\
\hline
\end{tabular}

Students might be surprised to see that the total cost approximates $\$ 70,000$. If they competed in a year round sport at a competitive level in high school, they might not be shocked by the per player cost. Case question 2 is intended to 
demonstrate how changes to certain variables impact the total cost. Case question 3 is intended to focus the students on the source of the biggest cost. The team pays $\$ 44,450$ to the club $(\$ 7500+\$ 15000+4500+1500+2250+2500$ $+\$ 4200+\$ 7000$ ), representing almost $70 \%$ of the team's budget for the year. Case question 4 should challenge students to identify tactical cost cutting solutions to stop the exodus of players to B and C level Premier Teams charging the same price but offering seemingly higher value. The students will have to make rational choices that won't significantly dilute the experience that players have become accustomed to expecting as a Select Team.

Note: Most of the information needed to prepare the budget is contained in the case. However, it might be necessary to provide the following table of inputs to simplify some of the calculations:

Table 2. Inputs

\begin{tabular}{l|r}
\hline Players & 15 \\
\hline Home Games & 12 \\
\hline Away Games & 12 \\
\hline Games & 24 \\
\hline Overnight Tournaments & 3 \\
\hline Local Tournaments & 5 \\
\hline Tournaments & 8 \\
\hline Hotel Costs per night & 200 \\
\hline Players per room & 2 \\
\hline Avg nights & 2 \\
\hline Winter games & 8 \\
\hline Winter Training - Indoor & 14 \\
\hline Winter Training - Outdoor & 14 \\
\hline Spring Training - Outdoor & 10 \\
\hline Indoor field Cost & 300 \\
\hline Outdoor Turf Cost & 250 \\
\hline
\end{tabular}

\section{AUTHOR BIOGRAPHIES}

Frederick C. Teufel, Jr., MBA, C.P.A., CGMA is in the accounting faculty of the Haub School of Business at Saint Joseph's University in Philadelphia, Pennsylvania and independent business consultant. He spent over 30 years in public accounting, initially in the Assurance practice at a Big 4 Accounting Firm and later as an Executive in the Financial Services Industry. Fred is a licensed C.P.A. in the State of Pennsylvania since 1985, and is a member of the AICPA and PICPA. He earned a BS in Accounting and MBA from Saint Joseph's University. Fred also earned his FINRA Series 27 and has served on the Boards of both Industry Groups as well as Non-Profits. (contact author: fteufel@sju.edu)

Joseph M. Larkin, Ph.D., C.P.A., is in the accounting faculty in the Haub School of Business at Saint Joseph's University in Philadelphia, Pennsylvania. He has published in the Journal of Business Case Studies, Journal of Business Ethics, Advances in Management Accounting, The Accounting Educators' Journal and Internal Auditing, as well as several practitioner journals. Dr. Larkin's teaching interests include Auditing and Managerial Accounting. Prior to entering academe, he worked for a major international public accounting firm in their audit practice.

\section{WHERE TO LEARN MORE}

Paul Keegan and Kate Santichen. How Soccer Bills Devoured This Family's Budget. Money Magazine, September 28, 2015. http:/time.com/money/4037391/soccer-bills-college-family-budget/

Immigrant Families Find Soccer Shockingly Expensive To Play In The U.S,. Transcript from NPR Morning Edition, Steve Inskeep - Host, May 26, 2017. https://www.npr.org/2017/05/26/530162575/immigrant-families-find-soccershockingly-expensive-to-play-in-the-u-s

Financial Management for Nonprofit Organizations: Policies and Practices - Ch 8 Developing Operating and Cash Budgets, pages 348 - 373

John Zietlow, Jo Ann Hankin, Alan Seidner, Tim O'Brien. Published by John Wiley and Sons (2018).

Business Plan - Buffalo Soccer Club. buffalosoccerclub.org/files/pdf/asfkbusinessplanv2.pdf 


\section{NOTES}

